Article

\title{
Noble-Metal Chalcogenide Nanotubes
}

\section{Nourdine Zibouche, Agnieszka Kuc, Pere Miró and Thomas Heine *}

School of Engineering and Science, Jacobs University Bremen, Campus Ring 1, 28759 Bremen, Germany; E-Mails: n.zibouche@jacobs-university.de (N.Z.); a.kuc@jacobs-university.de (A.K.); p.miro@jacobs-university.de (P.M.)

* Author to whom correspondence should be addressed; E-Mail: t.heine @ jacobs-university.de; Tel.: +49-421-200-3223.

External Editor: Reshef Tenne

Received: 9 May 2014; in revised form: 15 September 2014 / Accepted: 8 October 2014 /

Published: 24 October 2014

\begin{abstract}
We explore the stability and the electronic properties of hypothetical noble-metal chalcogenide nanotubes $\mathrm{PtS}_{2}, \mathrm{PtSe}_{2}, \mathrm{PdS}_{2}$ and $\mathrm{PdSe} \mathrm{e}_{2}$ by means of density functional theory calculations. Our findings show that the strain energy decreases inverse quadratically with the tube diameter, as is typical for other nanotubes. Moreover, the strain energy is independent of the tube chirality and converges towards the same value for large diameters. The band-structure calculations show that all noble-metal chalcogenide nanotubes are indirect band gap semiconductors. The corresponding band gaps increase with the nanotube diameter rapidly approaching the respective pristine 2D monolayer limit.
\end{abstract}

Keywords: noble-metal chalcogenides; nanotubes; strain energy; DFT

\section{Introduction}

Inorganic nanotubes (INTs) are a class of materials that are very attractive for many applications in nanotechnology due to their interesting physical and chemical properties, which arise from their low dimensionality. In 1930, Pauling had already stated that mismatched layered materials may form cylindrical shapes [1]. However, the first carbon-based tubular forms, namely carbon nanotubes (CNTs) have been observed by Iijima in 1991 [2]. At the same time, the synthesis of $\mathrm{WS}_{2}$ NTs by Tenne and co-workers [3], has demonstrated that these tubular systems are not limited to carbon, but can also be 
obtained from any other layered compound. Later on, several INTs have been prepared and produced such as transition-metal sulfides [3,4]. boron-nitrides [5,6], metal oxides [7,8], rare earth oxide [9] and more recently misfit layered compounds [10].

$\mathrm{WS}_{2}$ and $\mathrm{MoS}_{2}$ NTs, being the first synthesised INTs, are semiconductors. They have demonstrated excellent mechanical properties [11-16] and are known to be good solid lubricants [17]. They have also been suggested as scanning probe tips [18], catalysts [19], reinforcements for composite materials [20], photo-transistors [21], gas storage and host materials [22,23], etc. Later, other transition-metal chalcogenide (TMC) NTs have been reported such as $\mathrm{TiS}_{2}, \mathrm{NbS}_{2}, \mathrm{ReS}_{2}, \mathrm{TiSe}_{2}$ and $\mathrm{TaS}_{2}$ [24-28]. Subsequently, many more TMC NTs can be expected due to the large manifold of the layered TMC materials $[29,30]$. Nowadays, different techniques and strategies have been employed and developed for the synthesis and growth of large amount of NTs such as chemical transport technique [31], thermochemical decomposition [32] and in situ heating [33]. For example, $\mathrm{WS}_{2}$ and $\mathrm{MoS}_{2} \mathrm{NTs}_{\text {were }}$ produced using gas-solids reactions at high temperatures by the reduction of $\mathrm{WO}_{3}\left(\mathrm{MoO}_{3}\right)$ in the atmosphere of a mixture of $\mathrm{H}_{2}, \mathrm{~N}_{2}$ and $\mathrm{H}_{2} \mathrm{~S}$ gases [3,4,34].

In this work, we aim to extend the scope of inorganic nanotubular materials by investigating noble-metal chalcogenide $M X_{2}$ single wall nanotubes, where $M=\mathrm{Pt}$, $\mathrm{Pd}$ and $X=\mathrm{S}$, Se. Tubular forms based on these materials have not yet been experimentally observed, however, considering that $\mathrm{PtS}_{2}$, $\mathrm{PtSe}_{2}, \mathrm{PdS}_{2}, \mathrm{PdSe}_{2}$ belong to the family of layered TMCs, one can expect that such compounds may also form nanotubes. Structure and electronic structure of these noble metal chalcogenides have been subject to controversial debate in the 1950s and 1960s. Two possible phases of the Pd- and Pt-based TMCs were suggested, namely orthorhombic (pyrite) and 1T. [35-38]. We have calculated the relative stability of bulk and monolayered forms of these TMCs. While the orthorhombic phase is preferable for the bulk $\mathrm{PdS}_{2}$ material (energy difference of $56 \mathrm{meV}$ - all energies are given per $M X_{2}$ formula unit), the monolayers (MLs) favor the $1 T$ arrangement (energy difference of $68 \mathrm{meV}$ ). For the heavier $\mathrm{PdSe}_{2}$, we have found similar trends for the MLs, where the $1 T$ form is by $0.6 \mathrm{eV}$ more stable than the orthorhombic one. Bulk $\mathrm{PdSe}_{2}$ is unstable in the orthorhombic form, forbidding comparison with the $1 T$ structure. Therefore, the choice of the $1 T$ polytype to simulate the single-wall NTs is justified. In view of the renaissance of layered materials and the advance of experimental technology it is important to reexamine these phases and attempt exfoliation.

A recent study of bi- and monolayered noble-metal materials have shown interesting quantum confinement effects and electromechanical properties, suggesting them for applications in optoelectronics and flexible devices [39]. Therefore we have investigated, using density functional theory (DFT), the stability of the $M X_{2}$ NTs and their structural and electronic properties. The strain energy is found to be chirality independent and exhibits the characteristic dependence on the tube diameter $d$ $\left(\sim 1 / d^{2}\right)$. The band structure analysis shows that noble-metal chalcogenide NTs are all semiconducting in a similar way to their ML counterparts. Unlike $\mathrm{MoS}_{2}$ and $\mathrm{WS}_{2} \mathrm{NTs}$, where the band gap is direct and indirect for zigzag and armchair, respectively, $\mathrm{PtX}_{2}$ and $\mathrm{PdX}_{2}$ NTs have indirect band gaps which increase with the diameter. 


\section{Computational Details}

All calculations were carried out using density functional theory with the PBE (Perdew-Burke-Ernzerhof) exchange-correlation functional [40] as implemented in the Crystal09 code [41]. We have used an all electron triple-zeta valence basis set with one polarization function for sulphur atoms [42], a scalar-relativistic pseudopotential with 18 valence electrons for platinum atoms [43], Hay and Wadt effective core potentials with small core for palladium atoms [41], and the relativistic multi-electron pseudopotential with six valence electrons for selenium atoms [44]. We have employed helical boundary conditions, as implemented in the Crystal09 code [41], for the generation of the NT structures in order to reduce computational costs. Lattice vectors and atomic positions of $M X_{2}$ MLs and NTs were fully optimized. The tube diameters considered here are in the range of $10-50 \AA$, corresponding to chiral indices $(10,0)-(32,0)$ and $(6,6)-(24,24)$ for zigzag and armchair NTs, respectively. The shrinking factors of 16 for MLs and 8 for NTs were used, resulting in 30 and 5 $k$-points in the irreducible Brillouin zone, respectively, following the Monkhorst-Pack sampling [45]. Band structures were calculated along the high symmetry $k$-points using the $\mathrm{M}-\Gamma-\mathrm{K}-\mathrm{M}$ and $\mathrm{X}-\Gamma$ paths for MLs and NTs, respectively.

\section{Results and Discussion}

The monolayered noble-metal chalcogenides, considered here as large diameter NT limits, were adopted in the $1 T$ polymorph, with space group (P-3m1). Figure 1 shows the $1 T$ geometry for a monolayered structures in the left side, the top and the side views of zigzag and armchair NTs are represented in the middle and the right parts, respectively. The optimized lattice parameters and bond lengths with respect to the tube diameter are shown in Figure 2. Increasing the tube diameter $d$, the lattice vectors of the tubes decrease for both zigzag and armchair chiralities. For $(n, n)$ NTs, the lattice vectors correspond to those of the MLs; however, due to the curvature they enlarge as the diameters become smaller. Similar behaviour is observed for the bond lengths between metal and chalcogen atoms $(M-X)$, nevertheless, the convergence to the ML limit is much slower. Generally, the bond lengths and lattice vectors of selenide NTs are larger than those of sulphide NTs, and the same holds for the comparison of the platinum over the palladium forms.

The $M-X$ bond lengths can be divided into two types, the inner and the outer wall bond lengths, which are referred hereafter as $M-X_{i}$ and $M-X_{o}$, respectively. The $M-X_{o}\left(M-X_{i}\right)$ are longer (shorter) than the corresponding bond lengths found in the ML structures, and the deviations are more pronounced for armchair NTs than for zigzag NTs, particularly for small $d$. For diameters below $15 \AA$, the difference between outer and inner bond reaches $0.40 \AA$ and $0.25 \AA$ for armchair and zigzag NTs, respectively. These numbers strongly reduce to about $0.10 \AA$ difference for diameters of at least $40 \AA$. Recently, we have reported that the bond lengths of $\mathrm{MoS}_{2}$ and $\mathrm{WS}_{2}$ NTs exhibit opposite behaviour, where the zigzag NTs have longer (shorter) $M-X_{o}\left(M-X_{i}\right)$ bond lengths than the armchair NTs [46]. 
Figure 1. 1T 2D (monolayer) and 1D (tubular) forms of noble metal chalcogenides. The unit cell of 2D systems is shown.

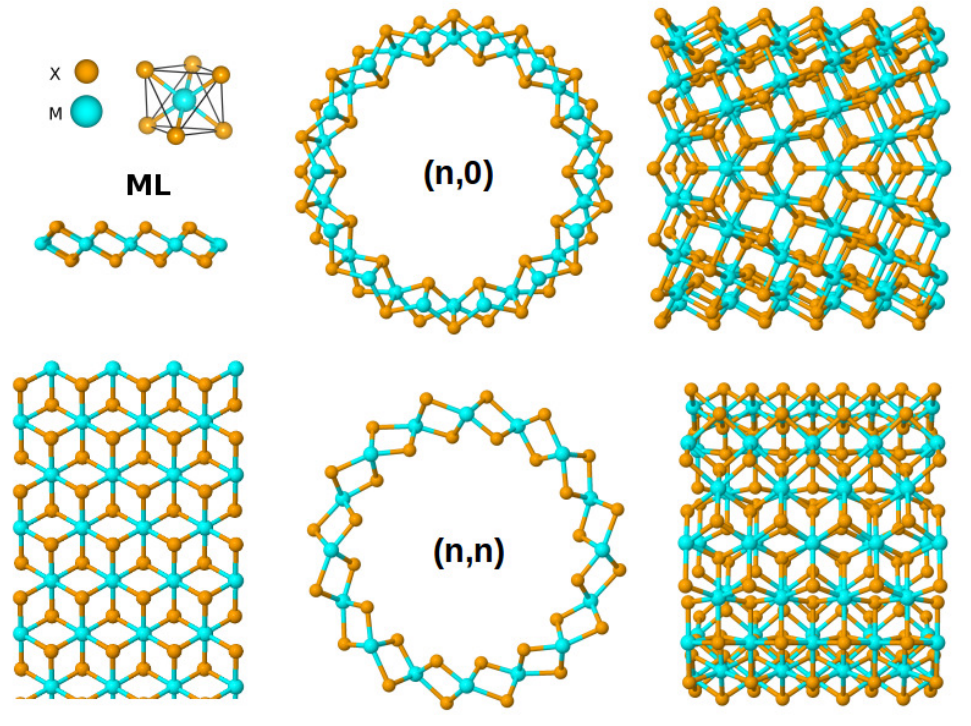

Figure 2. Lattice parameters and bond lengths $v s$. diameter of $M X_{2}$ NTs.
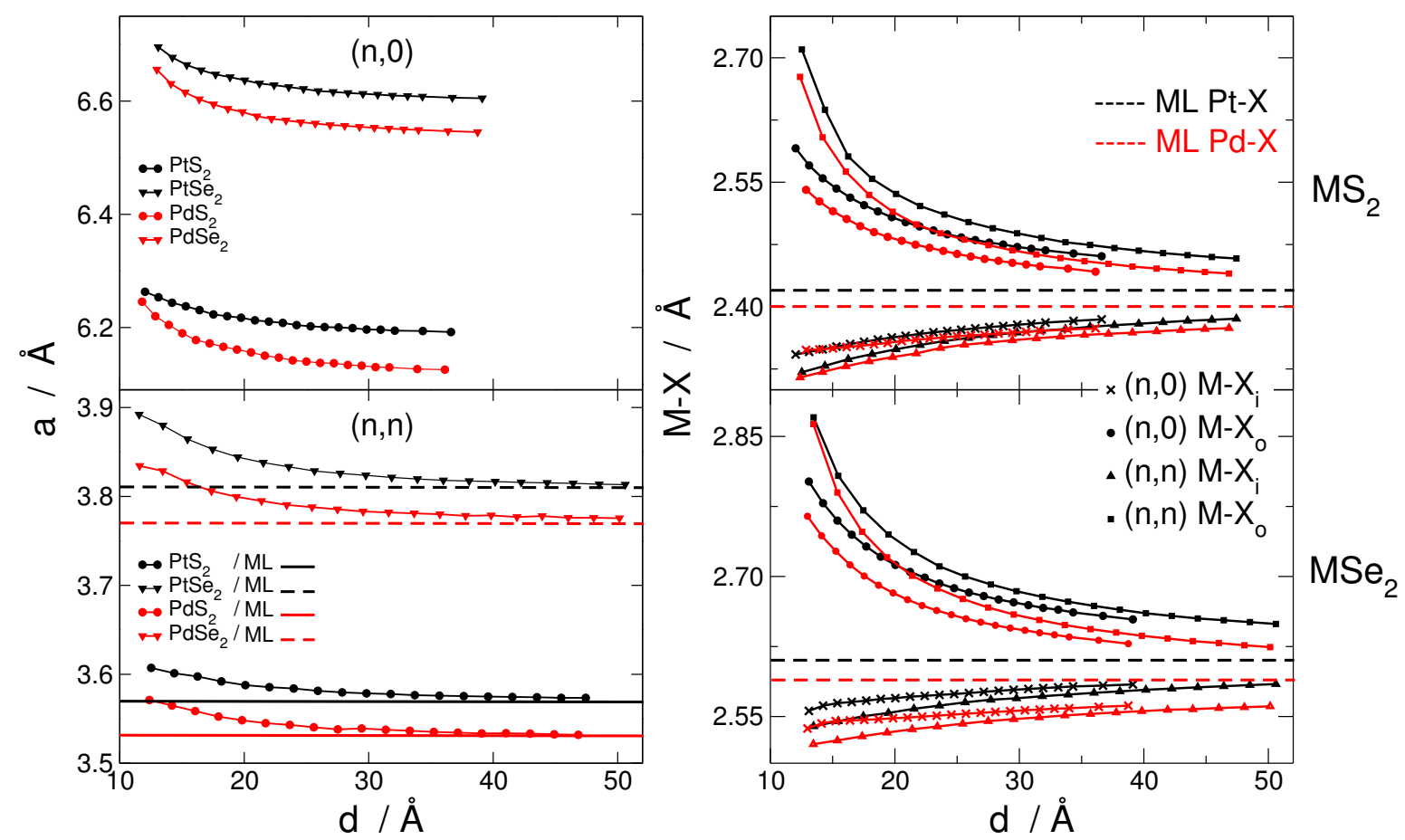

The stability of NTs can be expressed by the strain energy, $E_{\text {Strain }}$, which is the difference of total energy per atom of the tube and the respective ML. Generally, the strain energy of NTs is correlated to the tube diameter through the archetypal relation $E_{\text {Strain }} \sim 1 / d^{2}$. The calculated $E_{\text {Strain }}$ of $M X_{2}$ NTs with respect to their diameters (see Figure 3) follow the same dependence, where the strain energies decrease quadratically with the diameter and converge to the same value for all systems. The curves $E_{\text {Strain }}(d)$ were fitted to the equation $E_{\text {Strain }}=C / d^{2}$ with correlation coefficients greater than 0.999 and the values of coefficients $C$ are given in the Table 1 for each system. We note that strain energies of the $\operatorname{Pd} X_{2}$ NTs are smaller than for $\mathrm{Pt} X_{2}$ NTs for small diameters, this means that $\mathrm{Pd} X_{2}$ NTs are more stiff 
than $\mathrm{Pt} X_{2}$ NTs in that range. Furthermore, the strain energy of noble-metal chalcogenide tubes is the same for both zigzag and armchair chiralities, whereas for $\mathrm{MoS}_{2}$ and $\mathrm{WS}_{2}$ counterparts, armchair NTs are more stable than zigzag NTs, especially for large diameters [46]. In addition, the coefficients $C$ and the strain energy values of the noble-metal chalcogenide NTs are smaller than those of $\mathrm{MoS}_{2}$ and $\mathrm{WS}_{2}$ NTs. This means that noble-metal chalcogenide NTs are more favorable and easier to form than $\mathrm{MoS}_{2}$ and $\mathrm{WS}_{2}$ NTs.

Figure 3. Strain energy $v s$. diameter of $M X_{2}$ nanotubes.
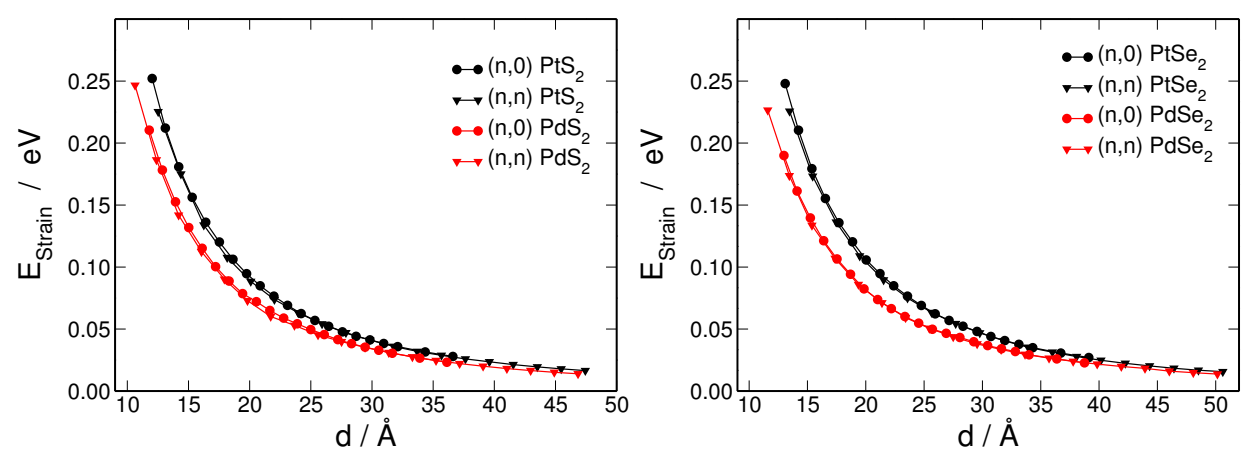

Table 1. Coefficients $C$ ( in $\mathrm{eV} \AA^{2}$ ) of the fitted curves $E_{\text {Strain }}=C / d^{2}$.

\begin{tabular}{ccc}
\hline System & Zigzag $(n, \mathbf{0})$ & Armchair $(n, n)$ \\
\hline $\mathrm{PtS}_{2}$ & 36.60 & 35.71 \\
$\mathrm{PtSe}_{2}$ & 42.47 & 41.22 \\
$\mathrm{PdS}_{2}$ & 29.71 & 28.46 \\
$\mathrm{PdSe}_{2}$ & 32.50 & 31.38 \\
$\mathrm{MoS}_{2}[46]$ & 57.50 & 50.90 \\
$\mathrm{WS}_{2}[46]$ & 58.14 & 59.68 \\
\hline
\end{tabular}

We have also investigated the electronic structure of these noble-metal chalcogenide systems. The $M X_{2}$ MLs are found to be semiconducting with indirect band gaps of $1.26 \mathrm{eV}, 1.75 \mathrm{eV}$, $0.74 \mathrm{eV}$ and $1.38 \mathrm{eV}$ for $\mathrm{PdS}_{2}, \mathrm{PtS}_{2}, \mathrm{PdSe}_{2}, \mathrm{PtSe}_{2}$, respectively. This is in agreement with the results of Miró et al. [39] for these $\mathrm{MX}_{2} \mathrm{MLs}$, where the obtained band gaps are $1.11 \mathrm{eV}, 1.75 \mathrm{eV}, 0.39 \mathrm{eV}$ and $1.05 \mathrm{eV}$ for $\mathrm{PdS}_{2}, \mathrm{PtS}_{2}, \mathrm{PdSe}_{2}$ and $\mathrm{PtSe}_{2}$, respectively, using similar level of theory.

The band gaps $\Delta$ versus the tube diameter of all $M X_{2}$ NTs are plotted in Figure 4. Similar to $\mathrm{MoS}_{2}$ and $\mathrm{WS}_{2}$ NTs, these band gaps of $M X_{2}$ NTs increase with the diameter and approach the band gaps of their respective MLs. For small $d$, the band gaps of armchair NTs become larger than zigazag NTs for all systems. Substituting Pd with Pt causes an increase in $\Delta$, while replacing $\mathrm{S}$ by $\mathrm{Se}$ decreases it. The band structures of noble-metal $M X_{2}$ MLs and NTs are depicted in Figure 5. Unlike $\mathrm{MoS}_{2}$ and $\mathrm{WS}_{2}$ MLs, where the band gaps are direct with values of 1.9 and $2.1 \mathrm{eV}$, respectively [47], the noble-metal MLs are all indirect bandgap semiconductors. This difference could be understood in the electronic configuration of the metal elements, as well as, in the $2 \mathrm{H}$ and $1 T$ symmetries of $\mathrm{MoS}_{2} / \mathrm{WS}_{2}$ and the noble-metal chalcogenides, respectively. The $M X_{2}$ chalcogenide NTs also exhibit indirect band gaps, for both zigazags and armchairs and for all systems, whereas $\mathrm{MoS}_{2}$ and $\mathrm{WS}_{2} \mathrm{NTs}$ show direct and indirect band gaps for zigzag and armchair forms, respectively [46]. 
Figure 4. Band gap vs. diameter of $M X_{2}$ nanotubes.
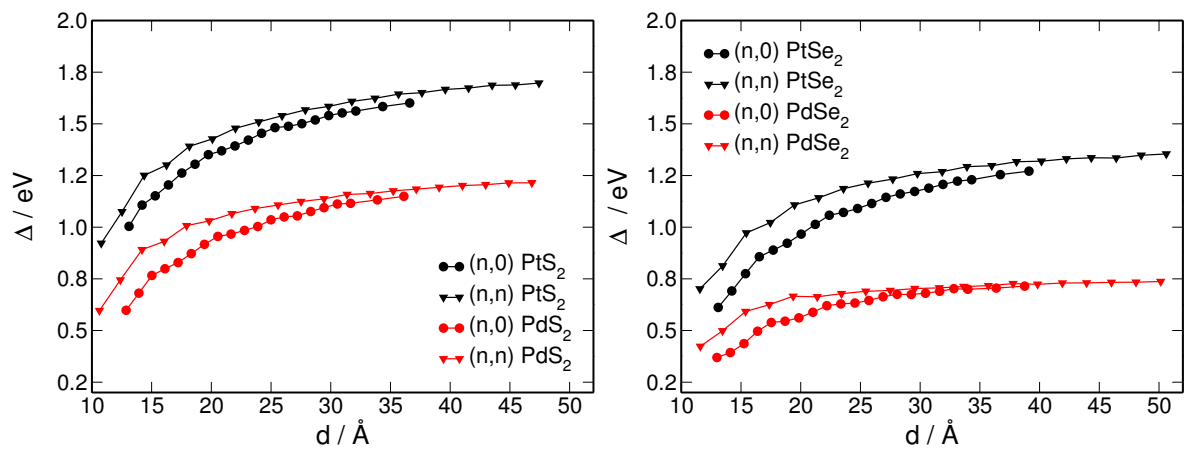

Figure 5. Band structures of monolayers, $(32,0),(18,18)$ nanotubes of $M X_{2}$ systems, respectively.
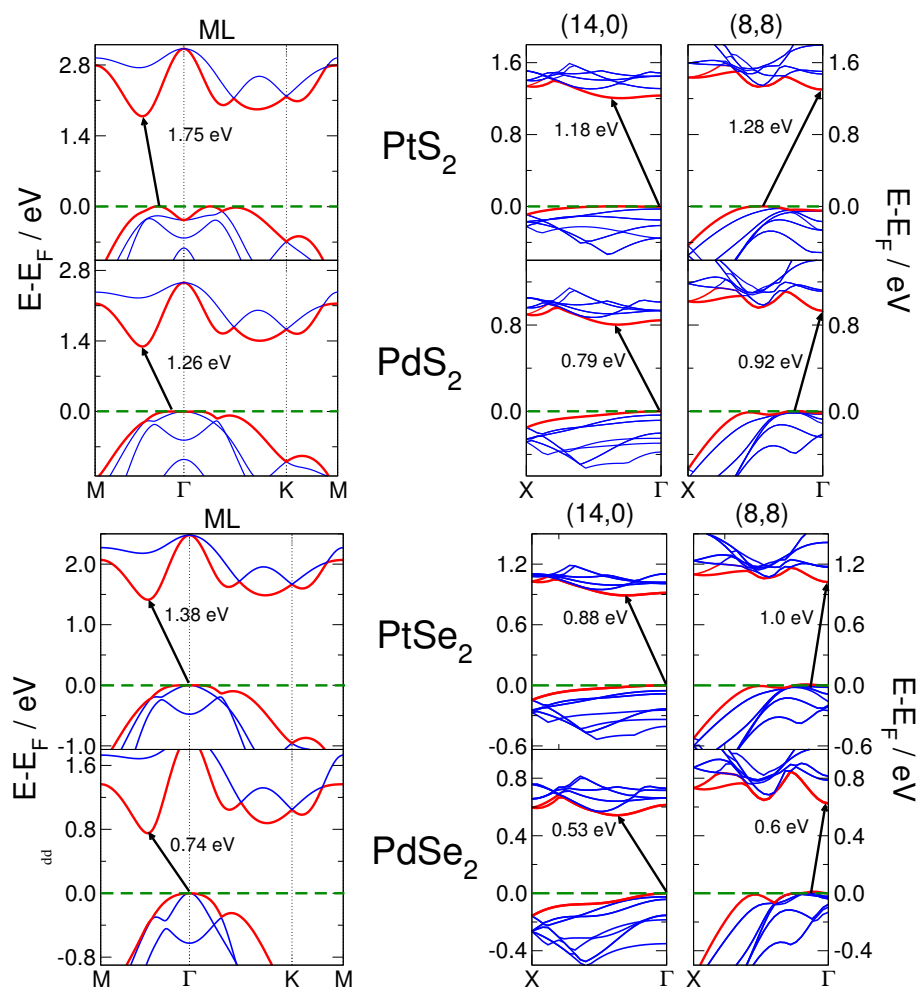

\section{Conclusions}

In analogy to the existing transition metal chalcogenide nanotubes, we have investigated hypothetical noble-metal chalcogenide nanotubes ( $\mathrm{PdS}_{2}, \mathrm{PdSe}_{2}, \mathrm{PtS}_{2}$ and $\left.\mathrm{PtSe}_{2} \mathrm{NTs}\right)$ through density functional theory calculations. We have shown that formation of these nanotubes is possible, since they have smaller strain energies than $\mathrm{MoS}_{2}$ or $\mathrm{WS}_{2}$ nanotubes. Furthermore, we have found that the strain energy of the studied nanotubes is chirality independent and decreases inverse quadratically with the tube diameter. Moreover, $\mathrm{Pd} X_{2}$ nanotubes are more stable than $\mathrm{Pt} X_{2}$ for nanotubes with small diameters. We have also examined the electronic structure of noble-metal chalcogenide monolayers and nanotubes, which are found to be all indirect band gap semiconductors in the ranges of $0.6-1.1 \mathrm{eV}(0.9-1.7 \mathrm{eV})$ and $0.3-0.8$ $\mathrm{eV}(0.6-1.3 \mathrm{eV})$ for $\mathrm{PdS}_{2}\left(\mathrm{PtS}_{2}\right)$ and $\mathrm{PdSe}_{2}\left(\mathrm{PtSe}_{2}\right) \mathrm{NTs}$, respectively. These NTs band gaps increase with the diameter rapidly approaching that of the respective pristine $2 \mathrm{D}$ monolayer. 


\section{Acknowldgements}

This work was supported by the German Research Council (Deutsche Forschungsgemeinschaft, HE 3543/18-1), the European Commission (FP7-PEOPLE-2009-IAPP QUASINANO, GA 251149 and FP7-PEOPLE-2012-ITN MoWSeS, GA 317451).

\section{Author Contributions}

Nourdine Zibouche, Agnieszka Kuc, Pere Miró and Thomas Heine generated, analyzed and discussed the results. Thomas Heine conceived this project. All authors contributed in writing this paper.

\section{Conflicts of Interest}

The authors declare no conflict of interest.

\section{References}

1. Pauling, L. The structure of the chlorites. Proc. Natl. Acad. Sci. USA 1930, 16, 578-582.

2. Iijima, S. Helical Microtubules of Graphitic Carbon. Nature 1991, 354, 56-58.

3. Tenne, R.; Margulis, L.; Genut, M.; Hodes, G. Polyhedral and Cylindrical Structures of Tungsten Disulfide. Nature 1992, 360, 444.

4. Margulis, L.; Salitra, G.; Tenne, R.; Talianker, M. Nested fullerene-like structures. Nature 1993, 365, 113-114.

5. Chopra, N.G.; Luyken, R.J.; Cherrey, K.; Crespi, V.H.; Cohen, M.L.; Louie, S.G.; Zettl, A. Boron Nitride Nanotubes. Science 1995, 269, 966-967.

6. Yin, L.W.; Bando, Y.; Golberg, D.; Li, M.S. Growth of Single-Crystal Indium Nitride Nanotubes and Nanowires by a Controlled-Carbonitridation Reaction Route. Adv. Mater. 2004, $16,1833-1838$.

7. Spahr, M.; Bitterli, P.; Nesper, R.; Mueller, M.; Krumeich, F.; Nissen, H.U. Redoxaktive Nanoröhren aus Vanadiumoxid. Angew. Chem. 1998, 110, 1339-1342.

8. Kasuga, T.; Hiramatsu, M.; Hoson, A.; Sekino, T.; Niihara, K. Titania Nanotubes Prepared by Chemical Processing. Adv. Mater. 1999, 11, 1307-1311.

9. Yada, M.; Mihara, M.; Mouri, S.; Kuroki, M.; Kijima, T. Rare Earth (Er, Tm, Yb, Lu) Oxide Nanotubes Templated by Dodecylsulfate Assemblies. Adv. Mater. 2002, 14, 309-313.

10. Radovsky, G.; Popovitz-Biro, R.; Stroppa, D.G.; Houben, L.; Tenne, R. Nanotubes from Chalcogenide Misfit Compounds: Sn-S and Nb-Pb-S. Acc. Chem. Res. 2014, 47, 406-416.

11. Zhu, Y.Q.; Sekine, T.; Brigatti, K.S.; Firth, S.; Tenne, R.; Rosentsveig, R.; Kroto, H.W.; Walton, D.R.M. Shock-Wave Resistance of $\mathrm{WS}_{2}$ Nanotubes. J. Am. Chem. Soc. 2003, 125, 1329-1333.

12. Kaplan-Ashiri, I.; Cohen, S.R.; Gartsman, K.; Rosentsveig, R.; Ivanovskaya, V.; Heine, T.; Seifert, G.; Wagner, H.D.; Tenne, R. Mechanical properties of individual $\mathrm{WS}_{2}$ nanotubes. Electron. Prop. Synth. Nanostruct. 2004, 723, 306-312. 
13. Kaplan-Ashiri, I.; Cohen, S.R.; Gartsman, K.; Rosentsveig, R.; Seifert, G.; Tenne, R. Mechanical behavior of individual $\mathrm{WS}_{2}$ nanotubes. J. Mater. Res. 2004, 19, 454-459.

14. Kaplan-Ashiri, I.; Cohen, S.R.; Gartsman, K.; Rosentsveig, R.; Ivanovskaya, V.; Heine, T.; Seifert, G.; Wagner, H.D.; Tenne, R. Direct tensile tests of individual $\mathrm{WS}_{2}$ nanotubes. Mater. Sci. Forum 2005, 475-479, 4097-4102.

15. Kaplan-Ashiri, I.; Cohen, S.R.; Gartsman, K.; Ivanovskaya, V.V.; Heine, T.; Seifert, G.; Wiesel, I.; Wagner, H.D.; Tenne, R. On the Mechanical Behavior of $\mathrm{WS}_{2}$ Nanotubes under Axial Tension and Compression. Proc. Natl. Acad. Sci. USA 2006, 103, 523-528.

16. Kaplan-Ashiri, I.; Tenne, R. Mechanical Properties of $\mathrm{WS}_{2}$ Nanotubes. J. Clust. Sci. 2007, 18, 549-563.

17. Rapoport, L.; Bilik, Y.; Feldman, Y.; Homyonfer, M.; Cohen, S.R.; Tenne, R. Hollow nanoparticles of $\mathrm{WS}_{2}$ as potential solid-state lubricants. Nature 1997, 387, 791-793.

18. Rothschild, A.; Cohen, S.R.; Tenne, R. $\mathrm{WS}_{2}$ nanotubes as tips in scanning probe microscopy. Appl. Phys. Lett. 1999, 75, 4025, doi:10.1063/1.125526.

19. Komarneni, M.R.; Yu, Z.; Burghaus, U.; Tsverin, Y.; Zak, A.; Feldman, Y.; Tenne, R. Characterization of Ni-Coated $\mathrm{WS}_{2}$ Nanotubes for Hydrodesulfurization Catalysis. Isr. J. Chem. 2012, 52, 1053-1062.

20. Zhang, W.; Ge, S.; Wang, Y.; Rafailovich, M.H.; Dhez, O.; Winesett, D.A.; Ade, H.; Shafi, K.V.; Ulman, A.; Popovitz-Biro, R. Use of functionalized $\mathrm{WS}_{2}$ nanotubes to produce new polystyrene/polymethylmethacrylate nanocomposites. Polymer 2003, 44, 2109-2115.

21. Unalan, H.E.; Yang, Y.; Zhang, Y.; Hiralal, P.; Kuo, D.; Dalal, S.; Butler, T.; Cha, S.N.; Jang, J.E.; Chremmou, K.; et al. ZnO Nanowire and $\mathrm{WS}_{2}$ Nanotube Electronics. IEEE Trans. Electron Devices 2008, 55, 2988-3000.

22. Wang, G.X.; Bewlay, S.; Yao, J.; Liu, H.K.; Dou, S.X. Tungsten Disulfide Nanotubes for Lithium Storage. Electrochem. Solid-State Lett. 2004, 7, A321-A323.

23. Hua, M.; Zhanliang, T.; Feng, G.; Jun, C.; Huatang, Y. Synthesis, characterization and hydrogen storage capacity of $\mathrm{MS}_{2}(M=\mathrm{Mo}$, Ti) nanotubes. Front. Chem. China 2006, 1, 260-263.

24. Chen, J.; Li, S.L.; Tao, Z.L.; Gao, F. Low-temperature synthesis of titanium disulfide nanotubes. Chem. Commun. 2003, 980-981.

25. Remskar, M.; Mrzel, A.; Jesih, A.; Leperevy, F. Metal-Alloyed $\mathrm{NbS}_{2}$ Nanotubes Synthesized by the Self-Assembly of Nanoparticles. Adv. Mater. 2002, 14, 680-684.

26. Coleman, K.S.; Sloan, J.; Hanson, N.A.; Brown, G.; Clancy, G.P.; Terrones, M.; Terrones, H.; Green, M.L.H. The Formation of $\mathrm{ReS}_{2}$ Inorganic Fullerene-like Structures Containing Re4 Parallelogram Units and Metal-Metal Bonds. J. Am. Chem. Soc. 2002, 124, 11580-11581.

27. Chen, J.; Tao, Z.L.; Li, S.L.; Fan, X.B.; Chou, S.L. Synthesis of $\mathrm{TiSe}_{2}$ Nanotubes/Nanowires. Adv. Mater. 2003, 15, 1379-1382.

28. Li, P.; Stender, C.L.; Ringe, E.; Marks, L.D.; Odom, T.W. Synthesis of $\mathrm{TaS}_{2}$ Nanotubes from $\mathrm{Ta}_{2} \mathrm{O}_{5}$ Nanotube Templates. Small 2010, 6, 1096-1099.

29. Miro, P.; Audiffred, M.; Heine, T. An Atlas of Two-Dimensional Materials. Chem. Soc. Rev. 2014, $43,6537-6554$. 
30. Nicolosi, V.; Chhowalla, M.; Kanatzidis, M.G.; Strano, M.S.; Coleman, J.N. Liquid Exfoliation of Layered Materials. Science 2013, 340, doi:10.1126/science.1226419.

31. Remskar, M.; Skraba, Z.; Regula, M.; Ballif, C.; Sanjines, R.; Levy, F. New crystal structures of $\mathrm{WS}_{2}$ : Microtubes, ribbons, and ropes. Adv. Mater. 1998, 10, 246-249.

32. Catherine, M.; Zelenski, P.K.D. Template Synthesis of Near-Monodisperse Microscale Nanofibers and Nanotubules of $\mathrm{MoS}_{2}$. J. Am. Chem. Soc. 1998, 120, 734-742.

33. Zhu, Y.Q.; Hsu, W.K.; Grobert, N.; Chang, B.H.; Terrones, M.; Terrones, H.; Kroto, H.W.; Walton, D.R.M. Production of $\mathrm{WS}_{2}$ Nanotubes. Chem. Mater 2000, 12, 1190-1194.

34. Feldman, Y.; Wasserman, E.; Srolovitz, D.J.; Tenne, R. High-Rate, Gas-Phase Growth of MoS ${ }_{2}$ Nested Inorganic Fullerenes and Nanotubes. Science 1995, 267, 222-225.

35. Gronvold, F.; Rost, E. On the sulfides, selenides and tellurides of palladium. Acta Chem. Scand. 1956, 10, 1620-1634.

36. Kjekshus, A.; Gronvold, F. High temperature X-ray study of the thermal expansionn of $\mathrm{PtS}_{2}, \mathrm{PtSe}_{2}$, $\mathrm{PtTe}_{2}$ and $\mathrm{PdTe}_{2}$. Acta Chem. Scand. 1959, 13, 1767-1774.

37. Gronvold, F.; Haraldsen, H.; Kjekshus, A. On the sulfides, selenides and tellurides of platinium. Acta Chem. Scand. 1960, 14, 1879-1893.

38. Wilson, J.A.; Yoffe, A.D. The Transition Metal Dichalcogenides Discussion and Interpretation of Observed Optical, Electrical and Structural Properties. Adv. Phys. 1969, 18, 193-335.

39. Miro, P.; Ghorbani-Asl, M.; Heine, T. Two Dimensional Materials Beyond $\mathrm{MoS}_{2}$ : Noble-Transition-Metal Dichalcogenides. Angew. Chem. Int. Ed. 2014, 53, 3015-3018.

40. Perdew, J.P.; Burke, K.; Ernzerhof, M. Generalized gradient approximation made simple. Phys. Rev. Lett. 1996, 77, 3865, doi:10.1103/PhysRevLett.77.3865.

41. Dovesi, R.; Saunders, V.R.; Roetti, R.; Orlando, R.; Zicovich-Wilson, C.M.; Pascale, F.; Civalleri, B.; Doll, K.; Harrison, N.M.; Bush, I.J.; et al. CRYSTAL09 User's Manual; University of Torino: Torino, Italy, 2009.

42. Peintinger, M.F.; Oliveira, D.V.; Bredow, T. Consistent Gaussian basis sets of triple-zeta valence with polarization quality for solid-state calculations. J. Comput. Chem. 2013, 34, 451-459.

43. Doll, K. Adsorption on the Pt surface a comparison of a gradient corrected functional and a hybrid functional. Surf. Sci. 2004, 573, $464-473$.

44. Stoll, H.; Metz, B.; Dolg, M. Relativistic energy-consistent pseudopotentials-recent developments. J. Comput. Chem. 2002, 23, 767-778.

45. Monkhorst, H.J.; Pack, J.D. Special Points For Brillouin-Zone Integrations. Phys. Rev. B 1976, 13, 5188, doi:10.1103/PhysRevB.13.5188.

46. Zibouche, N.; Kuc, A.; Heine, T. From layers to nanotubes: Transition metal disulfides $\mathrm{TMS}_{2}$. Eur. Phys. J. B 2012, 85, 49, doi:10.1140/epjb/e2011-20442-1.

47. Kuc, A.; Zibouche, N.; Heine, T. Influence of quantum confinement on the electronic structure of the transition metal sulfide $\mathrm{TS}_{2}$. Phys. Rev. B 2011, 83, doi:10.1103/PhysRevB.83.245213.

(C) 2014 by the authors; licensee MDPI, Basel, Switzerland. This article is an open access article distributed under the terms and conditions of the Creative Commons Attribution license (http://creativecommons.org/licenses/by/4.0/). 